\title{
Society for Research in Child Development \\ Wiley
}

Stylistic Variation at the "Single-Word" Stage: Relations between Maternal Speech

Characteristics and Children's Vocabulary Composition and Usage

Author(s): Julian M. Pine, Elena V. M. Lieven and Caroline F. Rowland

Source: Child Development, Vol. 68, No. 5 (Oct., 1997), pp. 807-819

Published by: Wiley on behalf of the Society for Research in Child Development

Stable URL: http://www.jstor.org/stable/1132034

Accessed: 20-10-2015 21:01 UTC

\section{REFERENCES}

Linked references are available on JSTOR for this article:

http://www.jstor.org/stable/1132034?seq=1\&cid=pdf-reference\#references_tab_contents

You may need to log in to JSTOR to access the linked references.

Your use of the JSTOR archive indicates your acceptance of the Terms \& Conditions of Use, available at http://www.jstor.org/page/ info/about/policies/terms.jsp

JSTOR is a not-for-profit service that helps scholars, researchers, and students discover, use, and build upon a wide range of content in a trusted digital archive. We use information technology and tools to increase productivity and facilitate new forms of scholarship. For more information about JSTOR, please contact support@jstor.org. 


\title{
Stylistic Variation at the "Single-Word" Stage: Relations between Maternal Speech Characteristics and Children's Vocabulary Composition and Usage
}

\author{
Julian M. Pine, Elena V. M. Lieven, and Caroline F. Rowland
}

\begin{abstract}
In this study we test a number of different claims about the nature of stylistic variation at the "single-word" stage by examining the relation between variation in early vocabulary composition, variation in early language use, and variation in the structural and functional properties of mothers' child-directed speech. Maternalreport and observational data were collected for 26 children at 10, 50, and 100 words. These were then correlated with a variety of different measures of maternal speech at 10 words. The results show substantial variation in the percentage of common nouns and unanalyzed phrases in children's early vocabularies, and significant relations between this variation and the way in which language is used by the child. They also reveal significant relations between the way in which mothers use language at 10 words and the way in which their children use language at 50 words and between certain formal properties of mothers' speech at 10 words and the percentage of common nouns and unanalyzed phrases in children's early vocabularies. However, most of these relations disappear when an attempt is made to control for possible effects of the child on the mother at Time 1. The exception is a significant negative correlation between mothers' tendency to produce speech that illustrates word boundaries and the percentage of unanalyzed phrases at 50 and 100 words. This suggests that mothers whose speech provides the child with information about where new words begin and end tend to have children with few unanalyzed phrases in their early vocabularies.
\end{abstract}

\section{INTRODUCTION}

It has been clear since the mid-1970s that there are substantial qualitative differences between children in their early "single-word" speech, with some children learning a high percentage of common nouns or object names in their first 50 and 100 words and others acquiring more heterogeneous vocabularies, often including a number of rote-learned or unanalyzed multiword phrases (Bates et al., 1994; Lieven, Pine, \& Dresner-Barnes, 1992; Nelson, 1973). These differences have often been found to be related to variation in maternal speech characteristics. Thus significant negative correlations have been found between referential style and maternal directiveness (Della Corte, Benedict, \& Klein, 1983; Nelson, 1973), and significant positive correlations have been found between referential style and maternal objectreferencing (Furrow \& Nelson, 1984; Klein, 1980); referential style and maternal common noun usage (Goldfield, 1993; Hampson, 1989; Klein, 1980); and referential style and maternal description of the immediate environment (Della Corte et al., 1983; Hampson, 1989). However, although it seems increasingly likely that some relation does exist between differences in mothers' interactional behavior and stylistic variation at the single-word level, the precise nature of this relation is still far from clear.

The present study represents an attempt to ad- dress this question by examining the relation between variation in children's early vocabulary composition, variation in children's early language use, and variation in the structural and functional properties of mothers' child-directed speech in a sample of 26 mother-infant dyads at approximately 10, 50, and 100 words. The central aim of the study is to test two alternative, although not necessarily mutually exclusive, models of the relation between variation in maternal speech characteristics and variation in children's single-word speech. The first of these models is the kind of functional similarity account proposed by Nelson (1981) and Della Corte et al. (1983). According to this view, variation in early vocabulary composition reflects differences in children's hypotheses about the function of language in social interaction based on differences in their experience of the way in which language is used by their parents. Thus, it is argued that children whose mothers tend to use language descriptively rather than directively tend to have a high percentage of nouns in their early vocabularies because they have acquired from their mothers a particular view of language as a tool for describing and categorizing as opposed to manipulating their environments. Such an account would seem to predict significant positive correla-

(c) 1997 by the Society for Research in Child Development, Inc. All rights reserved. 0009-3920/97/6805-0005\$01.00 
tions not only between maternal descriptiveness and children's noun learning but also between maternal descriptiveness and child descriptiveness and between maternal directiveness and child directiveness.

The second model is an account in terms of the interaction between children's processing mechanisms and the structure of the input to which they are exposed (Pine, 1994; Tomasello \& Todd, 1983). According to this view, variation in early vocabulary composition can be seen as a result of differences in the opportunities provided by different kinds of maternal utterances for the acquisition of particular kinds of vocabulary items. Thus, one plausible explanation for the relation between maternal descriptiveness and children's noun learning is that maternal descriptives tend to include nouns and also to model those nouns in ways that make it easy for the child to map them onto referents in the immediate environment. Such an account would predict that, rather than reflecting a general effect of maternal descriptiveness on children's noun learning, the relation between maternal descriptiveness and the percentage of nouns in children's early vocabularies should be specific to those maternal descriptives that themselves include nouns. That is to say, there should be a significant positive correlation between mothers' use of descriptives including nouns and children's noun learning, but no significant correlation between children's noun learning and mothers' use of descriptives that do not include nouns.

A plausible explanation for differences in the percentage of unanalyzed phrases in children's vocabularies, on the other hand, is that they might result from differences in the extent to which different kinds of maternal speech illustrate boundaries between words as opposed to boundaries between phrases, through the repetition and recombination of different lexical or phrasal elements (Peters, 1983, 1985). As far as we are aware, a direct test of this hypothesis has never been reported in the literature. However, it has often been argued that the redundancy of child-directed speech provides the child with a great deal of information about word and phrase boundaries (e.g., Ratner, 1996), and this has sometimes been found to be related to children's progress in grammatical development (e.g., HoffGinsberg, 1985). The present study therefore also includes an attempt to test the prediction that there will be a significant negative correlation between the percentage of frozen phrases in children's "single-word" vocabularies and the extent to which sequences of maternal utterances illustrate word boundaries by isolating, repeating, or recombining particular words or phrases.

\section{METHOD}

\section{Participants}

Participants in the study were 26 children of mixed SES whose parents had responded to an advertisement placed in the local newspaper. This included 14 girls and 12 boys and 12 first-borns and 14 laterborns. All of the children were from monolingual English-speaking families, and all were cared for primarily by their mothers, whose educational levels ranged from having left school at age 16 to having completed a university degree.

\section{Procedure}

The children were recruited for the study on the basis that they had maternal-report vocabularies of fewer than 10 words. Their progress was then monitored by administering a checklist over the telephone at approximately fortnightly intervals until the points at which mothers reported vocabularies of approximately 10, 50, and 100 words. At each of these points the children were visited at home. During these visits mothers were asked to fill in another vocabulary checklist, and a $40 \mathrm{~min}$ audio-recording was made of the children's spontaneous language use. This procedure was intended as a means of collecting vocabulary composition measures and spontaneous speech measures that were controlled as carefully as possible for vocabulary size at or around 10, 50, and 100 words (Lieven \& Pine, 1990; Pine \& Lieven, 1990). It inevitably resulted in the loss of data at some measurement points for some children. Thus, "10 word" measures are reported for only 24 children because two children (one girl and one boy) were found to have maternal-report vocabularies that were closer to 50 than to 10 words at the "10 word" recording session; "50 word" measures are reported for only 24 children because two children (one girl and one boy) were found to have maternal-report vocabularies that were closer to 100 than to 50 words at the "50 word" recording session; and " 100 word" measures are reported for only 22 children because one child (a girl) had been lost from the study by this stage, and three children (one girl and two boys) were found to have maternal-report vocabularies of 288,147 , and 161 words, respectively, at the "100 word" recording session. The children ranged in age from 0,9 to 1,6 at "10 words" ( $M=14.1$ months, $S D=2.6)$; from 1,1 
to 1,11 at "50 words" $(M=17.6$ months, $S D=2.8)$; and from 1,3 to 2,1 at " 100 words" ( $M=19.9$ months, $S D=2.8$ ). The children's maternal-report vocabularies ranged in size from 5 to 19 words at 10 words $(M=12.5, S D=3.7)$; from 41 to 74 words at 50 words $(M=53.8, S D=10.1)$; and from 92 to 129 words at 100 words $(M=108.7, S D=11.7)$.

\section{The Checklist}

The checklist was an Anglicized version of the vocabulary checklist section of the MacArthur Communicative Development Inventory (Infants) that had been modified in the following ways. First, because comprehension was not the focus of the present study, mothers were requested only to check off items that their child actually produced rather than items that she produced and / or comprehended. Second, because we were interested in collecting direct measures of the percentage of unanalyzed phrases as well as common nouns in children's early vocabularies, an additional multiword utterance checklist was appended to the main single-word checklist, and mothers were requested only to check off items on the single-word checklist if they had heard their child produce them as one-word utterances. The multiword checklist was made up of 49 phrases that had often been reported by mothers in diaries in the authors' previous research including the following: "Another one," "Don't do that," "Here you are," "I get it," "Sit down," and "What's that?" Finally, a section was added to the end of both the single-word and multiword checklists in which mothers were asked to write down any vocabulary items that they had heard their child produce but that were not listed on the checklist. This was intended as a means of collecting exhaustive maternal-report vocabularies that could be combined with additional vocabulary items from the observational data to provide vocabulary records that were as comprehensive as possible in the context of the present study (see Pine, Lieven, \& Rowland, 1996, for a more detailed discussion of this checklist).

\section{The Recording Session}

The 40 min audio-recording was based on two 20 min recording sessions, the first of which was a recording of the child at lunch and the second a recording of the child at play. These recordings were transcribed into a computerized database using the CHAT system from the CHILDES project (MacWhinney, 1991; MacWhinney \& Snow, 1985, 1990) and used to derive measures of the mothers' language use at 10 words and the children's language use at 10, 50, and 100 words. They were also used to augment the maternal-report vocabulary records by adding in vocabulary items found on the tapes that had not been reported by the mothers to give combined maternalreport + observational measures of vocabulary composition. The criterion for inclusion of items from the tapes was that they were used spontaneously by the child (i.e., had not been modeled by another speaker within a previous $10 \mathrm{~s}$ time frame) and were used with a clearly identifiable meaning.

\section{Child Language Measures}

Vocabulary composition measures. Vocabulary composition measures were obtained by classifying all of the items in the combined maternal-report + observational 10, 50, and 100 word vocabularies into one of the following categories: common nouns, onomatopoeic words, proper nouns, interactive words, other words, frozen phrases, intermediate utterances, and constructed utterances, using the coding scheme set out in Lieven et al. (1992).

The reliability of this classification was assessed by having a second coder independently classify all the items in four children's 100 word vocabularies. Reliability expressed as percentage agreement was $99.2 \%$ (kappa $=0.99)$.

Observational measures of vocabulary composition and usage. Observational measures of vocabulary composition and usage were obtained by classifying all of the children's utterances on the 10,50, and 100 word tapes (excluding direct imitations, self-repetitions, and unintelligible or partially intelligible utterances) into the same categories as the vocabulary data. The observational vocabulary composition measures were then used to assess the validity of the checklist vocabulary composition measures by examining the relation between checklist and observational measures of the percentage of common nouns and frozen phrases at 50 and 100 words. The results of this analysis revealed significant correlations for both common nouns and frozen phrases at both 50 and 100 words, $r(22)=0.56, p<.01$, and $r(22)=0.53, p<.01$, respectively at 50 words, and $r(20)=0.64, p<.01$, and $r(20)$ $=0.45, p<.05$, respectively, at 100 words, and are consistent with previous research which shows that maternal diary records can represent a valid and reliable source of information about children's early vocabulary development (e.g., Dale, Bates, Reznick, \& Morisset, 1989; Fenson et al., 1994; Snyder, Bates, \& Bretherton, 1981). It is perhaps worth noting, how- 
ever, that, although statistically significant, these correlations are fairly modest in size, suggesting that it may be a mistake to treat maternal-report and observational measures of vocabulary composition as if they were more or less equivalent (see Pine et al., 1996, for a more detailed discussion of this issue).

Functional measures of children's speech. Functional measures of children's speech were obtained by classifying all of the children's utterances on the 10, 50, and 100 word tapes (excluding direct imitations, selfrepetitions, and unintelligible or partially intelligible utterances) into one of the following categories: elicited responses, conversational responses, attention, demand, protest, labeling, description, and miscellaneous, using the coding scheme set out in Pine (1992a). Given our reliance on audio- rather than video-recorded data, an "unclassifiable" category was also included and used for cases where there was insufficient information on the tape for a coding decision to be made (e.g., cases where it was unclear whether a child was labeling or demanding an object). In practice, however, the frequency of such cases was relatively low (i.e., less than $3 \%$ on average at both 50 and 100 words), because demands tended to be uttered with a different intonation from labels and descriptions and to be followed up with more urgent self-repetitions if they did not result in the desired response.

The reliability of the functional classification was assessed by having a second coder independently code three 50 word and three 100 word tapes. Reliability expressed as percentage agreement was $89.3 \%$ $($ kappa $=0.88)$.

\section{Maternal Language Measures}

Measures of the percentage of common nouns per utterance in mothers' speech. Measures of the percentage of common nouns per utterance in mothers' speech were obtained by dividing the total number of common nouns that occurred in the mother's childdirected speech at 10 words (excluding false starts, utterances read directly from books, and unintelligible or partially intelligible utterances) by the total number of child-directed utterances that the mother produced and multiplying by 100 .

The reliability of these measures was assessed by having a second coder independently code all of the utterances on four 10 word transcripts for the presence of common nouns. Reliability expressed as percentage agreement was $99 \%$ (kappa $=0.96)$.

Functional measures of mothers' speech. Functional measures of mothers' speech were obtained by classifying all of the child-directed maternal utterances on the 10 word tapes (excluding false starts, utterances read directly from books, and unintelligible or partially intelligible utterances) into one of the following categories: attentional directives, behavioral directives, tutorial prompts, requests for information, description of the environment, and other, using the coding scheme set out in Pine (1992b).

The reliability of this classification was assessed by having a second coder independently code four 10 word transcripts. Reliability expressed as percentage agreement was $86.5 \%$ (kappa $=0.83$ ).

Measures of the segmentability of mothers' speech. Measures of the segmentability of mothers' speech were obtained by coding each successive maternal utterance on the 10 word tapes, first, for whether it was a single- or multiword utterance and, second, for whether it shared lexical material with any of the previous five maternal utterances in the discourse, provided that they occurred within a $10 \mathrm{~s}$ time frame. Utterances were coded as "isolating" utterances if they were single-word utterances or if the repetition of lexical items or strings of lexical items in the same position as in a previous utterance illustrated the break between a single word and the frame in which it occurred. This meant that single words could be isolated in any of the following ways: (1) by being produced as a single word utterance (e.g., "Dog"); (2) by being repeated in two different sentence frames (e.g., "That's a dog" followed by "Look at the dog"); or (3) by being used to fill the slot in a repeated sentence frame (e.g., "That's a cat" followed by "That's a dog").

The reliability of these measures was assessed by having a second coder independently code four 10 word transcripts. Reliability expressed as percentage agreement was 91\% (kappa $=0.88)$.

\section{RESULTS}

\section{Variation in Vocabulary Composition}

In this section we report results for the combined maternal report + observational measures of vocabulary composition at 50 and 100 words. Descriptive statistics for these measures are presented in Table 1 from which it can be seen that there is quite substantial variation between children in the percentage of vocabulary items falling into all of the categories of the coding scheme. However, what is particularly noteworthy in the context of the present study is the variation found in the percentage of common nouns and frozen phrases in the children's 50 and 100 word vocabularies. Thus, although common nouns make up an average of around $45 \%$ of the words in the chil- 
Table 1 Descriptive Statistics for the Vocabulary Composition Measures at $\mathbf{5 0}$ and $\mathbf{1 0 0}$ Words

\begin{tabular}{lrrc}
\hline Measures & $M$ & $S D$ & Range \\
\hline 50 word: & & & \\
$\quad$ Total vocabulary size $(N)$ & 65.5 & 13.1 & $46-94$ \\
Common nouns (\%) & 42.5 & 12.4 & $14.9-74.1$ \\
Onomatopoeic words (\%) & 7.9 & 4.7 & $1.2-17.2$ \\
Proper nouns (\%) & 10.0 & 4.4 & $3.4-8.9$ \\
Interactive words (\%) & 12.7 & 4.2 & $5.2-19.6$ \\
Other words (\%) & 17.9 & 5.2 & $8.6-25.8$ \\
Frozen phrases (\%) & 9.3 & 7.3 & $0-26.9$ \\
Potentially productive utter- & & & \\
$\quad$ ances (N) & 2.3 & 2.9 & $0-10$ \\
100 word: & & & \\
$\quad$ Total vocabulary size (N) & 132.9 & 17.5 & $103-168$ \\
Common nouns (\%) & 46.1 & 7.2 & $32.3-59.6$ \\
$\quad$ Onomatopoeic words (\%) & 6.1 & 2.8 & $.7-12.5$ \\
Proper nouns (\%) & 9.0 & 3.9 & $1.9-15.7$ \\
$\quad$ Interactive words (\%) & 8.8 & 2.2 & $4.5-14.6$ \\
Other words (\%) & 19.8 & 4.9 & $8.7-29.2$ \\
Frozen phrases (\%) & 10.1 & 5.4 & $.6-18.3$ \\
Potentially productive utter- & & & \\
$\quad$ ances (N) & 7.7 & 8.6 & $0-34$ \\
\hline
\end{tabular}

dren's vocabularies, scores on this measure range from $14.9 \%$ to $74.1 \%$ at 50 words and from $32.3 \%$ to $59.6 \%$ at 100 words. Similarly, although frozen phrases make up an average of around $10 \%$ of the words in the children's vocabularies, scores on this measure range from 0 to $26.9 \%$ at 50 words and from 0.6 to $18.3 \%$ at 100 words. This variation is consistent with that reported by Lieven et al. (1992) using the same coding scheme, although the average percentage of common nouns is somewhat higher $(M=$ $42.5 \%$ versus $M=33.2 \%$ and $M=46.1 \%$ versus $M$ $=37.5 \%$ at 50 and 100 words, respectively) and the average percentage of frozen phrases somewhat lower $(M=9.3 \%$ versus $M=17.8 \%$ and $M=10.1 \%$ versus $M=21.2 \%$ at 50 and 100 words, respectively).

Differences between the pairs of 50 and 100 word measures were analyzed using correlated $t$ tests. These revealed significant increases between 50 and 100 words in the percentage of common nouns, $M=$ 42.0 versus $M=45.9, t(19)=3.50, p<.01$, the percentage of frozen phrases, $M=7.8$ versus $M=9.8$, $t(19)=3.16, p<.01$, and the percentage of other words, $M=18.0$ versus $M=20.5, t(19)=2.78, p<$ .05 , and significant decreases in the percentage of onomatopoeic words, $M=8.8$ versus $M=6.2, t(19)=$ $4.85, p<.001$, the percentage of proper nouns, $M=$ 10.4 versus $M=8.6, t(19)=1.73, p<.05$, and the percentage of interactive words, $M=12.9$ versus $M$ $=8.8, t(19)=6.04, p<.001$. These results are consistent with the findings of several other recent studies of early lexical development which suggest that there are systematic developmental changes in the relative numerical importance of different kinds of words in young children's vocabularies (e.g., Bates et al., 1994; Lieven et al., 1992; Pine \& Lieven, 1990). They therefore underline the importance of controlling vocabulary size when attempting to measure stylistic variation in children's early vocabulary composition.

\section{Relation between Variation in Vocabulary Composition and Variation in Vocabulary Size}

In this section we report results for the relation between the 50 and 100 word measures of vocabulary composition and the corresponding measures of vocabulary size. It will be remembered that the need to control the size of the vocabularies on which vocabulary composition measures are based was one of the principal motivations for the data collection procedure adopted in the present study. However, it can be seen from Table 1 that, despite this procedure, there was still quite substantial variation between children in the size of their vocabularies. This reflects the fact that, although our methodology was quite successful in eliciting maternal-report measures controlled for vocabulary size at or around the 50 and 100 word levels, the inclusion of additional vocabulary items from the 10,50, and 100 word recording sessions led to quite substantial increases in the size of some of the children's vocabularies and, hence, to an increase in the range of the scores on this particular measure.

The relation between variation in vocabulary size and variation on the different vocabulary composition measures was therefore examined further using Pearson product moment coefficients. No significant correlations were found between vocabulary size and the percentage of common nouns, frozen phrases, onomatopoeic words, proper nouns or other words at either 50 or 100 words. However, there was a significant negative correlation between vocabulary size and the percentage of interactive words in the children's vocabularies at both 50 and 100 words, $r$ (22) $=-.41, p<.05$, and $r(20)=-.51, p<.05$, respectively. This suggests that the procedure adopted in the present study was not entirely successful in eliminating nonstylistic variation in vocabulary composition attributable to differences in children's current level of vocabulary development. However, given that there were significant developmental changes between 50 and 100 words on all of the vocabulary composition measures, it would seem to have resulted in measures of stylistic variation that are reasonably free of such contamination. 
Stability of Vocabulary Composition Measures over Time and Their Relation to Variation in Rate of Vocabulary Development

In this section we report results for the relation between variation in vocabulary composition and variation in rate of vocabulary development and results for the relation between variation in vocabulary composition at 50 and 100 words. These issues were investigated, first, by correlating all of the measures in Table 1 with age at 50 and 100 words and, second, by correlating measures of vocabulary composition based on the first 50 words with measures of vocabulary composition based on the second 50 words (i.e., those words acquired between the 50 and 100 word recording sessions). The first of these analyses revealed no significant correlations between any of the vocabulary composition measures and age at 50 or 100 words. The second revealed significant correlations for common nouns, $r(18)=.45, p<.05$, and frozen phrases, $r(18)=.66, p<.01$, but no significant correlations for any of the other vocabulary composition measures. These findings replicate those of Lieven et al. (1992) and suggest that the percentages of common nouns and frozen phrases in children's vocabularies are not only important dimensions of variation at 50 and 100 words but are also unrelated to differences in rate of language acquisition and show some stability over time.

Relation between Variation in Vocabulary Composition and Variation in Productive Multiword Speech

In this section we report results for the relation between variation in vocabulary composition and variation in children's productive multiword speech. This question was investigated by correlating the percentages of common nouns and frozen phrases in children's 50 and 100 word vocabularies with the number of utterances coded as potentially productive (i.e., intermediate or constructed) at 50 and 100 words. Spearman rank order correlations were used in this analysis because several children had no potentially productive utterances at either 50 or 100 words. These revealed significant positive correlations between the percentage of frozen phrases and the number of potentially productive utterances at 50 and 100 words, $\rho=0.77, N=24, p<.01$, and $\rho=$ $0.75, N=22, p<.01$, respectively, and a significant negative correlation and a near-significant negative correlation between the percentage of common nouns and the number of potentially productive utterances at 50 and 100 words, $\rho=-0.43, N=24$, $p<.05$, and $\rho=-0.41, N=22, p<.10$, respectively.
These findings also replicate those of Lieven et al. (1992) and are consistent with the idea that "phrasal" children may be building limited scope patterns around phrases initially learned as unanalyzed wholes at lower vocabulary levels than children whose vocabularies consist primarily of single words.

\section{Variation in Vocabulary Composition and Variation in Language Use}

In this section we report results for the 50 and 100 word measures of children's language use and the relation between these measures and the 50 and 100 word measures of vocabulary composition. Descriptive statistics for the functional measures of children's speech at 50 and 100 words are presented in Table 2 . It should be noted that the response category scores represent percentages of the children's total spontaneous speech, whereas the initiation category scores represent percentages of the children's total spontaneous initiations.

As with the vocabulary composition measures, it is clear that there is substantial variation between children in the percentage of utterances falling into all of the categories of the coding scheme. The question of the stability of this variation over time was addressed by correlating the pairs of 50 and 100 word initiation measures. Significant positive correlations were found for the statement and miscellaneous categories, $r(18)=.55, p<.01$, and $r(18)=.85, p<.01$, respectively, and near-significant correlations for the regulation, protest, and labeling categories, $r(18)=$ $.38, p<.10, r(18)=.36, n s$, and $r(18)=.37, n s$, respectively. These results suggest that variation in the way in which language is used by individual children does show some stability over time, and they are therefore consistent with the idea that there is stylistic variation not only in the kind of words that children are acquiring early in development but also in the way in which these words are used.

The question of the relation between this variation and variation in vocabulary composition was investigated by correlating all of the initiation categories presented in Table 2 with the percentage of common nouns and frozen phrases at 50 and 100 words. Significant positive correlations were found between common nouns at 50 and 100 words and the percentage of statement and labeling initiations, $r(22)=.46$, $p<.05$, and $r(20)=.62, p<.01$ for statement initiations, and $r(22)=.43, p<.05$, and $r(20)=.72, p<$ .01 for labeling, respectively; significant negative correlations between common nouns at 50 and 100 words and the percentage of miscellaneous initiations, $r(22)=-.64, p<.01$, and $r(20)=-.71, p<$ 
Table 2 Descriptive Statistics for the Functional Measures of Children's Speech at 50 and 100 Words

\begin{tabular}{|c|c|c|c|c|}
\hline \multirow[b]{2}{*}{ Children's Speech Measures } & \multicolumn{2}{|c|}{$\begin{array}{l}50 \text { Word } \\
\text { Measures }\end{array}$} & \multicolumn{2}{|c|}{$\begin{array}{l}100 \text { Word } \\
\text { Measures }\end{array}$} \\
\hline & $M$ & $S D$ & $M$ & $S D$ \\
\hline Total utterances $(N)$ & 61.4 & 36.5 & 112.7 & 51.9 \\
\hline Elicited responses (\%) & 13.8 & 11.0 & 21.4 & 14.4 \\
\hline Conversational responses (\%) & 8.6 & 8.7 & 11.5 & 6.7 \\
\hline Total initiations $(N)$ & 47.3 & 29.5 & 75.0 & 35.5 \\
\hline Attention $(\%)$ & 12.3 & 12.9 & 12.1 & 8.5 \\
\hline Regulation (demand + protest) $(\%)$ & 17.6 & 10.8 & 19.5 & 9.9 \\
\hline Demand $(\%)$ & 13.2 & 7.2 & 14.4 & 8.8 \\
\hline Protest $(\%)$ & 4.5 & 5.7 & 5.2 & 4.9 \\
\hline Statement (labeling + description) $(\%)$ & 50.2 & 22.3 & 50.7 & 16.3 \\
\hline Labeling (\%) & 29.1 & 20.8 & 22.1 & 12.6 \\
\hline Description (\%) & 21.2 & 8.9 & 28.6 & 11.3 \\
\hline Miscellaneous (\%) & 17.8 & 16.7 & 14.7 & 13.6 \\
\hline Unclassifiable (\%) & 2.4 & 3.1 & 2.9 & 3.1 \\
\hline
\end{tabular}

.01 , respectively; and significant positive correlations between frozen phrases at 50 and 100 words and the percentage of miscellaneous initiations, $r(22)=.49, p$ $<.05$, and $r(20)=.50, p<.05$. There was also a significant negative correlation between common nouns at 100 words and the child's use of utterances to manipulate attention, $r(20)=-.51, p<.05$, and a significant negative correlation between frozen phrases at 100 words and the child's use of utterances to reject or protest about undesired activities or events, $r(20)$ $=-.45, p<.05$.

In contrast to the findings of Pine (1992a), these results provide quite strong support for the view that there is a positive relation between noun learning and labeling. However, they fail to provide any evidence for a positive relation between phrase learning and regulation. Indeed, the only significant correlation between the percentage of frozen phrases and any of the regulation categories (i.e., that between frozen phrases and protest at 100 words), was in the opposite direction to that predicted. On the other hand, the percentage of frozen phrases in children's vocabularies was significantly positively related to the miscellaneous initiations category at both 50 and 100 words. This suggests that, rather than concentrating on the regulative as opposed to the referential functions of language, phrasal children tend to use language in ways that do not fit easily into a referential-regulative dichotomy.

\section{Variation in Maternal Speech Acts}

In this section we report results for the 10 word maternal speech act measures. Descriptive statistics for these measures are presented in Table 3. It should be noted that despite wide variation in the age of the children at this measurement point, there were no significant correlations between any of these measures and the children's age at 10 words.

The question of the interrelation between the maternal speech act measures was investigated using Pearson product moment correlations. Significant negative correlations were found between the percentage of descriptives and the percentage of attentional directives in mothers' speech and the percentage of descriptives and the percentage of behavioral directives, $r(22)=-.44, p<.05$, and $r(22)=.45, p<$ .05 , respectively. There was also a significant positive correlation between the percentage of attentional directives and the percentage of requests for information, $r(22)=.53, p<.05$. However, there was no significant correlation between the percentage of attentional directives and the percentage of behavioral directives, $r(22)=.14, n s$, nor indeed between any of the other speech act measures.

This pattern of correlations is consistent with the view that the extent to which mothers use language to describe aspects of the environment rather than to direct their children's activity is an important dimension of variation at this point in development, and hence with the idea that it may be possible to explain referential-regulative differences in children's early language use in terms of some kind of functional similarity effect. However, it is perhaps worth noting that it is also consistent with recent work showing that directiveness is not a unitary dimension of maternal behavior. This presumably reflects the fact that behavioral directives can be, and often are, used by 
Table 3 Descriptive Statistics for the Maternal Speech Measures at 10 Words

\begin{tabular}{lrrr}
\hline Maternal Speech Measures & $M$ & \multicolumn{1}{c}{$S D$} & Range \\
\hline Total utterances (N) & 396.5 & 176.4 & $83-834$ \\
Attentional directives (\%) & 2.8 & 2.3 & $.1-10.5$ \\
Behavioral directives (\%) & 20.6 & 7.1 & $10.5-35.6$ \\
Tutorial prompts (\%) & 10.4 & 4.3 & $2.8-20.9$ \\
Requests for information (\%) & 10.8 & 5.1 & $3.4-26.3$ \\
Description of the environment (\%) & 20.1 & 6.7 & $9.6-36.5$ \\
$\quad$ Descriptives including nouns (\%) & 10.1 & 4.6 & $3.1-21.5$ \\
$\quad$ Descriptives not including nouns (\%) & 10.0 & 3.0 & $5.5-17.5$ \\
Common nouns per utterance (\%) & 26.3 & 8.1 & $14.8-42.9$ \\
Utterances illustrating word boundaries (\%) & 52.3 & 8.0 & $27.7-64.2$ \\
\hline
\end{tabular}

mothers to respond to as well as to direct the child's current focus of attention (e.g., Akhtar, Dunham, \& Dunham, 1991; Hoff-Ginsberg, 1991; Pine, 1992b).

\section{Variation in the Content and Segmentability of} Maternal Speech

In this section we report results for the 10 word measures of the content and segmentability of maternal speech. Descriptive statistics for these measures are presented in Table 3. It should be noted that despite wide variation in the age of the children at this measurement point, there were no significant correlations between either of these measures and the children's age at 10 words. However, significant positive correlations were found between total maternal speech and the percentage of nouns per utterance and between total maternal speech and the percentage of utterances illustrating word boundaries, $r(22)$ $=.45, p<.05$, and $r(22)=.53, p<.05$, respectively. Moreover, there was also a significant positive correlation between the percentage of nouns per utterance and the percentage of maternal descriptives, $r(22)=$ $.42, p<.05$, and a significant positive correlation between the percentage of utterances illustrating word boundaries and the percentage of tutorial prompts, $r(22)=.48, p<.05$.

This pattern of correlations is consistent with the view that descriptive language use by mothers tends to result in a higher percentage of common nouns in their child-directed speech. However, it also suggests that more talkative mothers tend to produce a higher percentage of common nouns and a higher percentage of utterances illustrating word boundaries. This last finding can be partly explained by the fact that mothers who produce relatively few child-directed utterances are likely to obtain low scores on the word boundary measure simply because their utterances tend to be more broadly spaced than mothers who produce a lot of child-directed utterances and are hence more likely to fall within different $10 \mathrm{~s}$ time frames. However, given that two of the characteristic features of child-directed speech are its repetitiveness and its limited semantic range, it probably also reflects the fact that the more utterances mothers produce, the more repetitive their language tends to become and, hence, the more likely they are to produce partial repetitions of their own previous utterances (Hoff-Ginsberg, 1994).

Relation between Variation in Maternal Speech and Stylistic Variation in Children's Language

In this section we report results for the relation between the maternal 10 word measures and the child 50 and 100 word measures. This relation was investigated by correlating all of the maternal language measures with the percentage of common nouns and frozen phrases, and the percentage of statement and regulation initiations at 50 and 100 words. The results of this analysis are presented in Table 4 and seem to provide some support both for a functional similarity and for a structural account of the relation between variation in maternal and child speech. Thus, there were no significant correlations between maternal descriptiveness nor maternal behavioral directiveness and either of the vocabulary composition measures. However, as predicted by the structural account, there was a significant correlation between descriptives including nouns and the percentage of common nouns at 50 although not 100 words, $r(20)$ $=.45, p<.05$, and $r(19)=.21, n s$, respectively, and between the word boundary measure and the percentage of frozen phrases at 50 and 100 words, $r$ (20) $=-.47, p<.05$, and $r(19)=-.61, p<.01$, respectively.

On the other hand, as predicted by the functional similarity account, there was a significant correlation between maternal descriptiveness and the percentage of statement initiations in children's speech at 50 
Table 4 Correlations between Maternal Speech Measures at 10 Words and the Percentage of Common Nouns and Frozen Phrases and Statement and Regulation Initiations at 50 and 100 Words

\begin{tabular}{lcccc}
\hline & $\begin{array}{c}\text { Common } \\
\text { Nouns }\end{array}$ & $\begin{array}{c}\text { Frozen } \\
\text { Phrases }\end{array}$ & $\begin{array}{c}\text { Statement } \\
\text { Initiations }\end{array}$ & $\begin{array}{c}\text { Regulation } \\
\text { Initiations }\end{array}$ \\
\hline Child language measures at 50 words: & & & & \\
Total utterances $(N)$ & .20 & $-.45^{*}$ & .31 & -.07 \\
Attentional directives (\%) & $-.50^{*}$ & .36 & -.33 & .05 \\
Behavioral directives (\%) & -.12 & .00 & -.28 & .31 \\
Tutorial prompts (\%) & -.11 & -.35 & .12 & -.08 \\
Requests for information (\%) & -.30 & $.50^{*}$ & -.17 & .03 \\
Description of the environment (\%) & .35 & -.07 & $.50^{*}$ & -.25 \\
Descriptives including nouns (\%) & $.45^{*}$ & -.13 & .36 & .00 \\
Descriptives not including nouns (\%) & .10 & .03 & $.54^{* *}$ & $-.53^{*}$ \\
Common nouns per utterance (\%) & .37 & -.08 & .10 & .35 \\
Utterances illustrating word boundaries (\%) & .13 & $-.47^{*}$ & .25 & -.16 \\
Child language measures at 100 words: & & & & .37 \\
Total utterances ( $N$ ) & .11 & $-.45^{*}$ & .26 & .14 \\
Attentional directives (\%) & .17 & .19 & -.01 & .12 \\
Behavioral directives (\%) & -.03 & .22 & -.19 & -.42 \\
Tutorial prompts (\%) & -.18 & -.30 & .23 & $.47^{*}$ \\
Requests for information (\%) & -.03 & .03 & -.33 & -.22 \\
Description of the environment (\%) & .10 & -.33 & .29 & -.06 \\
Descriptives including nouns (\%) & .21 & -.41 & .31 & -.39 \\
Descriptives not including nouns (\%) & -.07 & -.14 & .19 & .22 \\
Common nouns per utterance (\%) & .24 & -.38 & .24 & -.05 \\
Utterances illustrating word boundaries (\%) & .19 & $-.61^{* *}$ & .43 & \\
\hline
\end{tabular}

${ }^{*} p<.05 ;{ }^{* *} p<.01$, two-tailed.

words, $r(20)=.50, p<.05$, although no significant correlation at 100 words, $r(19)=.29, n s$, and no significant correlation between maternal directiveness and the percentage of regulative initiations in children's speech at either 50 or 100 words, $r(20)=.31$, $n s$, and $r(19)=.12, n s$, respectively.

The fact that these results suggest specific relations between both the formal and the functional properties of mothers' and children's speech underlines the importance of distinguishing between formal and functional levels of analysis when analyzing the relation between maternal and child language. However, it is important to note that there were also some relatively high, and in some cases significant, correlations between the maternal 10 word measures and the equivalent child 10 word measures, and between the child 10 word measures and the child 50 and 100 word measures (see Table 5). This raises the possibility that at least some of the relations reported in Table 4 may be mediated by indirect effects due to relations between maternal and child language at Time 1 and child language at Time 1 and Time 2 (Richards, 1994; Yoder \& Kaiser, 1989). The above analysis was therefore repeated with the relevant relations between the maternal and child 10 word measures partialed out. That is to say, for each relation between a child measure at 50 or 100 words and a maternal measure at
10 words, a partial correlation was computed with the relation between the corresponding child measure at 10 words and the same maternal measure at 10 words partialed out.

Partialing Out Possible Effects of the Child at Time 1 on the Mother at Time 1

In this section we report results for the relation between the maternal 10 word measures and the child 50 and 100 word measures after partialing out possible effects of the child at Time 1 on the mother at Time 1 . The results of this analysis are presented in Table 6, from which it can be seen that there are only five significant correlations that survive the partialing procedure. These are a significant negative correlation between the word boundary measure and frozen phrases at 50 words, $r(19)=-.47, p<.05$; a significant negative correlation between the word boundary measure and frozen phrases at 100 words, $r(18)=-.64, p<.01$; a significant positive correlation between descriptives not including nouns and statement initiations at 50 words, $r(19)=.44, p<.05$; a significant negative correlation between descriptives not including nouns and regulation initiations at 50 words, $r(19)=-.54, p<.05$, and a significant negative correlation between tutorial prompts and regula- 
Table 5 Correlations between Child Language Measures at 10 Words and Maternal Speech Measures at 10 Words and Child Language Measures at 50 and 100 Words

\begin{tabular}{lcccc}
\hline & \multicolumn{3}{c}{ Child Language Measures at 10 Words } \\
\cline { 2 - 5 } Maternal Speech Measures & $\begin{array}{c}\text { Common } \\
\text { Nouns }\end{array}$ & $\begin{array}{c}\text { Frozen } \\
\text { Phrases }\end{array}$ & $\begin{array}{c}\text { Statement } \\
\text { Initiations }\end{array}$ & $\begin{array}{c}\text { Regulation } \\
\text { Initiations }\end{array}$ \\
\hline Total utterances (N) & .33 & -.22 & .26 & .01 \\
Attentional directives (\%) & -.35 & $.45^{*}$ & -.28 & .01 \\
Behavioral directives (\%) & .05 & .08 & -.04 & -.26 \\
Tutorial prompts (\%) & -.29 & -.07 & .11 & .05 \\
Requests for information (\%) & $-.42^{*}$ & .19 & $-.48^{*}$ & $.43^{*}$ \\
Description of the environment (\%) & $.42^{*}$ & -.20 & $.54^{* *}$ & .14 \\
Descriptives including nouns (\%) & $.42^{*}$ & -.23 & $.47^{*}$ & .20 \\
Descriptives not including nouns (\%) & .30 & -.11 & $.48^{*}$ & .01 \\
Common nouns per utterance (\%) & .26 & -.23 & .21 & .24 \\
Utterances illustrating word boundaries (\%) & .17 & -.18 & .26 & .14 \\
Common nouns at 50 words (\%) & $.60^{* *}$ & $-.59^{* *}$ & .19 & .13 \\
Frozen phrases at 50 words (\%) & $-.50^{*}$ & $.58^{* *}$ & -.09 & .09 \\
Statement initiations at 50 words (\%) & .42 & -.23 & .41 & -.03 \\
Regulation initiations at 50 words (\%) & -.14 & -.32 & -.26 & .31 \\
Common nouns at 100 words (\%) & $.45^{*}$ & $-.49^{*}$ & .07 & .26 \\
Frozen phrases at 100 words (\%) & -.22 & $.56^{* *}$ & .00 & -.18 \\
Statement initiations at 100 words (\%) & .29 & -.38 & .32 & .19 \\
Regulation initiations at 100 words (\%) & .29 & -.21 & -.16 & .23 \\
\hline
\end{tabular}

${ }^{*} p<.05 ;{ }^{* *} p<.01$, two tailed.

Table 6 Partial Correlations between Maternal Speech Measures at 10 Words and the Percentage of Common Nouns and Frozen Phrases and Statement and Regulation Initiations at 50 and 100 Words

\begin{tabular}{lcccc}
\hline Maternal Language Measures at 10 Words & $\begin{array}{c}\text { Common } \\
\text { Nouns }\end{array}$ & $\begin{array}{c}\text { Frozen } \\
\text { Phrases }\end{array}$ & $\begin{array}{c}\text { Statement } \\
\text { Initiations }\end{array}$ & $\begin{array}{c}\text { Regulation } \\
\text { Initiations }\end{array}$ \\
\hline Child language measures at 50 words: & & & & \\
Total utterances $(N)$ & -.02 & -.42 & .22 & -.08 \\
Attentional directives (\%) & -.42 & .16 & -.28 & .01 \\
Behavioral directives (\%) & -.14 & .07 & -.27 & .42 \\
Tutorial prompts (\%) & .11 & -.35 & .09 & -.10 \\
Requests for information (\%) & -.13 & .41 & -.01 & -.17 \\
Description of the environment (\%) & .14 & .00 & .37 & -.28 \\
Descriptives including nouns (\%) & .28 & -.05 & .22 & -.04 \\
Descriptives not including nouns (\%) & -.10 & .06 & $.44^{*}$ & $-.54^{*}$ \\
Common nouns per utterance (\%) & .30 & .07 & .04 & .31 \\
Utterances illustrating word boundaries (\%) & .03 & $-.47^{*}$ & .21 & -.25 \\
Child language measures at 100 words: & & & & .34 \\
Total utterances ( $N$ ) & -.02 & -.43 & .19 & .34 \\
Attentional directives (\%) & .30 & .10 & .08 & .23 \\
Behavioral directives (\%) & -.06 & .19 & -.19 & .18 \\
Tutorial prompts (\%) & .06 & -.42 & .24 & $-.46^{*}$ \\
Requests for information (\%) & .13 & .11 & -.21 & .43 \\
Description of the environment (\%) & -.14 & -.28 & .15 & -.28 \\
Descriptives including nouns (\%) & -.03 & -.35 & .18 & -.16 \\
Descriptives not including nouns (\%) & -.24 & -.10 & .04 & -.40 \\
Common nouns per utterance (\%) & .08 & -.27 & .17 & .14 \\
Utterances illustrating word boundaries (\%) & .15 & $-.64^{* *}$ & .44 & -.12 \\
\hline
\end{tabular}

${ }^{*} p<.05 ;{ }^{* *} p<.01$, two-tailed. 
tion initiations at 100 words, $r(18)=-.46, p<.05$. There were, however, also several near-significant correlations, some of which are at least consistent with a functional similarity account, including a near-significant positive correlation between maternal descriptiveness and statement initiations at 50 words, $r(19)=.37, p<.10$, and a near-significant positive correlation between maternal directiveness and regulation initiations at 50 words, $r(19)=.42, p<.10$.

These results provide quite strong support for the view that it may be possible to explain variation in early phrase learning in terms of variation in the information about word boundaries that is available to young children in their input. They also provide some evidence, albeit rather weak, for an effect of variation in mothers' language use on variation in children's language use, although any such effect seems to have disappeared by 100 words and to be unrelated to variation in early vocabulary composition at either 50 or 100 words. However, they do not seem to provide any evidence for an effect of variation in maternal speech characteristics on children's early noun learning, at least once relations between maternal and child language at Time 1 have been partialed out. This suggests that the effects reported in previous research may have been due to effects of the child on the mother's behavior at Time 1 rather than to effects of the mother's behavior at Time 1 on the child's development between Time 1 and Time 2, and underlines the need for future studies to control for such indirect effects when investigating the relation between variation in maternal speech characteristics and variation in children's early vocabulary composition.

\section{DISCUSSION}

The major purpose of the present study was to test two alternative models of the relation between variation in maternal speech characteristics and variation in children's single-word speech: a functional similarity account and an account in terms of the interaction between children's processing mechanisms and the structure of the input to which they are exposed. The results seem to provide some support for both of these models, although there was a failure to replicate previous findings of relations between the percentage of common nouns in children's early vocabularies and maternal descriptiveness, maternal directiveness, and maternal common noun usage. This suggests that it is possible to find specific relations between both the formal and the functional properties of mothers' and children's speech and, hence, underlines the importance of distinguishing between formal and functional levels of analysis when analyzing relations between maternal and child language. However, when an attempt was made to control for effects of the child on the mother's behavior at Time 1 , very few significant correlations remained. Indeed, no significant correlations were found between any of the maternal speech variables and the percentage of common nouns at either 50 or 100 words, although there were significant negative correlations between the word boundary measure and the percentage of frozen phrases at both 50 and 100 words; and nearsignificant positive correlations between maternal descriptiveness and statement initiations at 50 although not 100 words, and between maternal directiveness and regulation initiations at 50 although not 100 words.

These results seem to provide some evidence, albeit rather weak, for an effect of variation in mothers' language use on variation in children's language use, although any such effect seems to have disappeared by 100 words and to be unrelated to variation in early vocabulary composition at either 50 or 100 words. However, they also suggest that previous findings of apparent effects of maternal speech characteristics on children's noun learning (e.g., Pine's, 1994, finding of a specific relation between descriptives, including nouns, and the percentage of nouns in children's first 50 words), may actually reflect effects of the child's behavior on the mother rather than effects of the mother's behavior on the child. Of course, this is not to say that there are no direct effects of mothers' interactional behavior on children's noun learning, nor indeed that the variables measured in the present study may not exert effects at earlier points in development. However, the fact that there are already significant relations between maternal behavior at 10 words and children's language at 10 words, and between children's language at 10 words and children's language at 50 words, does tend to suggest that children's noun-learning preferences may be set rather early in development and be relatively unaffected by subsequent variation in maternal speech characteristics, at least of the kind examined in the present study.

These results contrast sharply with the results for frozen phrases which were found to be significantly negatively correlated with the word boundary measure at both 50 and 100 words, even after partialing. This provides quite strong support for the view that there is a relation between variation in children's tendency to acquire unanalyzed phrases early in development and variation in the information about word boundaries that is available to them in their input. This seems to us to be quite an important finding be- 
cause it is, as far as we are aware, the first to show a link between some property of maternal speech and the child's tendency to acquire unanalyzed phrases early in development. In the past this tendency has tended to be explained in terms of hypothetical differences in children's processing mechanisms or abilities. However, the results of the present study suggest that it may be possible to explain it in terms of the interaction between processing mechanisms that are common to all children and differences in the structure of the input to which they are exposed.

Given that these differences seem to be related to differences in the amount of child-directed speech that mothers produce, it is possible that phrase learning in early vocabulary development is actually a kind of default strategy adopted by children whose input is less overtly tutorial in nature and perhaps, as a result, less rich in information about how utterance strings can be segmented into words. This suggestion is consistent with Heath's (1983) description of the early language of Trackton children who, despite receiving very little child-directed speech during the early stages of acquisition, appeared to break into productive language after an initial period in which they repeated fragments of the speech that they heard around them. It is also consistent with Pine's (1995) finding of a significant correlation between the proportion of unanalyzed phrases in second-born children's first 100 words and the size of the age difference between these children and their older siblings. This suggests that the acquisition of unanalyzed phrases in early vocabulary development increases as the sibling-directed speech overheard by laterborns increases in complexity relative to their own current language level. If this interpretation is correct, it implies that phrase learning in early vocabulary development may be an important strategy for many children, particularly those who do not receive finely tuned input (Lieven, 1994). Moreover, given that Pine and Lieven (1993) have demonstrated continuity between the phrases in children's early vocabularies and the structures that underlie their early multiword speech, it may also be that the acquisition of a large number of phrases in early vocabulary development may provide such children with an alternative route into productivity (Peters, 1983).

\section{ACKNOWLEDGMENTS}

We would like to thank all the families who took part in the research reported here. We would also like to thank Brian MacWhinney and two anonymous reviewers for their constructive comments on an earlier draft of this article. This research was funded by the
Economic and Social Research Council, Grant No. R000234221.

\section{ADDRESSES AND AFFILIATIONS}

Corresponding author: Julian M. Pine, Department of Psychology, University of Nottingham, NG7 2RD, United Kingdom; e-mail: jp@psyc.nott.ac.uk. Elena V. M. Lieven is at the University of Manchester; and Caroline F. Rowland is at the University of Nottingham.

\section{REFERENCES}

Akhtar, N., Dunham, F., \& Dunham, P. J. (1991). Directive interactions and early vocabulary development: The role of joint attentional focus. Journal of Child Language, 18, 41-49.

Bates, E., Marchman, V., Thal, D., Fenson, L., Dale, P., Reznick, J. S., Reilly, J., \& Hartung, J. (1994). Developmental and stylistic variation in the composition of early vocabulary. Journal of Child Language, 21, 85-123.

Dale, P., Bates, E., Reznick, J., \& Morisset, C. (1989). The validity of a parent report instrument of child language at twenty months. Journal of Child Language, 16, 239-249.

Della Corte, M., Benedict, H., \& Klein, D. (1983). The relationship of pragmatic dimensions of mothers' speech to the referential-expressive distinction. Journal of Child Language, 10, 35-43.

Fenson, L., Dale, P. S., Reznick, J. S., Bates, E., Thal, D. J., \& Pethick, S. J. (1994). Variability in early communicative development. Monographs of the Society for Research in Child Development, 59(5, Serial No. 242).

Furrow, D., \& Nelson, K. (1984). Environmental correlates of individual differences in language acquisition. Journal of Child Language, 11, 523-534.

Goldfield, B. A. (1993). Noun bias in maternal speech to one-year-olds. Journal of Child Language, 20, 85-99.

Hampson, J. (1989). Elements of style: Maternal and child contributions to referential and expressive styles of language acquisition. Unpublished doctoral dissertation, City University of New York.

Heath, S. B. (1983). Ways with words: Language, life and work in communities and classrooms. Cambridge: Cambridge University Press.

Hoff-Ginsberg, E. (1985). Some contributions of mothers' speech to their children's syntactic growth. Journal of Child Language, 12, 367-385.

Hoff-Ginsberg, E. (1991). Mother-child conversation in different social classes and communicative settings. Child Development, 62, 782-796.

Hoff-Ginsberg, E. (1994). Influences of mother and child on maternal talkativeness. Discourse Processes, 18, 105-117.

Klein, D. (1980). Expressive and referential communication in children's early language development: Their relationship to mothers' communicative styles. Unpublished doctoral dissertation, Michigan State University. 
Lieven, E. V. M. (1994). Crosslinguistic and crosscultural aspects of language addressed to children. In C. Gallaway \& B. J. Richards (Eds.), Input and interaction in language acquisition (pp. 74-106). Cambridge: Cambridge University Press.

Lieven, E. V. M., \& Pine, J. M. (1990). Review of "From first words to grammar: Individual differences and dissociable mechanisms" by E. Bates, I. Bretherton \& L. Snyder. Journal of Child Language, 17, 495-501.

Lieven, E. V. M., Pine, J. M., \& Dresner-Barnes, H. (1992). Individual differences in early vocabulary development: Redefining the referential-expressive distinction. Journal of Child Language, 19, 287-310.

MacWhinney, B. (1991). The CHILDES project. Hillsdale, NJ: Erlbaum.

MacWhinney, B., \& Snow, C. (1985). The Child Language Data Exchange System. Journal of Child Language, 12, 271-296.

MacWhinney, B., \& Snow, C. (1990). The Child Language Data Exchange System: An update. Journal of Child Language, 17, 457-472.

Nelson, K. (1973). Structure and strategy in learning to talk. Monographs of the Society for Research in Child Development, 38(1-2, Serial No. 149).

Nelson, K. (1981). Individual differences in language development: Implications for development and language. Developmental Psychology, 17, 170-187.

Peters, A. M. (1983). The units of language acquisition. Cambridge: Cambridge University Press.

Peters, A. M. (1985). Language segmentation: Operating principles for the perception and analysis of language. In D. I. Slobin (Ed.), The crosslinguistic study of language acquisition (pp. 1029-1064). Hillsdale, NJ: Erlbaum.

Pine, J. M. (1992a). The functional basis of referential style: Evidence from children's spontaneous speech. First Language, 12, 39-55.

Pine, J. M. (1992b). Maternal style at the early one-word stage: Re-evaluating the stereotype of the directive mother. First Language, 12, 169-186.
Pine, J. M. (1994). Environmental correlates of lexical variation: Interactional style and the structure of the input. Applied Psycholinguistics, 15, 357-372.

Pine, J. M. (1995). Variation in vocabulary development as a function of birth order. Child Development, 66, 272-281.

Pine, J. M., \& Lieven, E. V. M. (1990). Referential style at thirteen months: Why age-defined cross-sectional measures are inappropriate for the study of strategy differences in early language development. Journal of Child Language, 17, 625-631.

Pine, J. M., \& Lieven, E. V. M. (1993). Reanalysing rotelearned phrases: Individual differences in the transition to multi-word speech. Journal of Child Language, 20, 551571.

Pine, J. M., Lieven, E. V. M., \& Rowland, C. F. (1996). Observational and checklist measures of vocabulary composition: What do they mean? Journal of Child Language, 23, 573-589.

Ratner, N. B. (1996). From "signal to syntax": But what is the nature of the signal? In J. L. Morgan \& K. Demuth (Eds.), Signal to syntax: Bootstrapping from speech to grammar in early acquisition (pp. 135-150). Mahwah, NJ: Erlbaum.

Richards, B. J. (1994). Child-directed speech and influences on language acquisition: Methodology and interpretation. In C. Gallaway \& B. J. Richards (Eds.), Input and interaction in language acquisition (pp. 74-106). Cambridge: Cambridge University Press.

Snyder, L., Bates, E., \& Bretherton, I. (1981). Content and context in early lexical development. Journal of Child Language, 8, 565-582.

Tomasello, M., \& Todd, J. (1983). Joint attention and lexical acquisition style. First Language, 4, 197-212.

Yoder, P., \& Kaiser, A. (1989). Alternative explanations for the relationship between maternal verbal interaction style and child language development. Journal of Child Language, 16, 141-160. 